\title{
CARBON NANOTUBE BASED POLYVINYLALCOHOL-POLYVINYLPYROLIDONE NANOCOMPOSITE HYDROGELS FOR CONTROLLED DRUG DELIVERY APPLICATIONS
}

\author{
Bengi ÖZKAHRAMAN ${ }^{1}$, Emel TAMAHKAR IRMAK ${ }^{2, *}$ \\ ${ }^{1}$ Department of Polymer Engineering, Faculty of Engineering, Hitit University, Çorum, Turkey \\ ${ }^{2}$ Department of Chemical Engineering, Faculty of Engineering, Hitit University, Çorum, Turkey
}

\begin{abstract}
Controlled drug release systems present a significant alternative to the conventional drug dosages providing drug release for prolonged time periods. Nanocomposite hydrogels offer an important potential for drug release with enhanced physicochemical properties. In this study, the preparation of carbon nanotube (CNT)-based Polyvinylalcohol-Polyvinylpyrolidone (PVA/PVP) nanocomposite hydrogels namely, CNT-25, CNT-50 and CNT-100 were succeedded via the freeze/thawing method with the addition of different amounts of CNT. The nanocomposite hydrogels were characterized by swelling tests, SEM, FTIR, DSC and BET measurements. It was determined that CNT-50 was the most suitable hydrogel for drug release studies having better morphological properties with homogenous distribution of CNT throughout the polymeric nanocomposite matrix. The release of 5-fluororacil (5-FU) as a model drug was investigated in-vitro. The release of 5-FU from CNT-based PVA/PVP nanocomposite hydrogels was exhibited controlled release for one week at $\mathrm{pH}$ 7.4. The amount of released 5-FU was effectively increased with the addition of CNT into the hydrogel matrix. Korsmeyer-Peppas model was well fitted for determining the release mechanism of 5-FU from CNT-based PVA/PVP nanocomposite hydrogels corresponding the combination of diffusion of the drug and the dissolution of polymer chains.
\end{abstract}

Keywords: Drug release systems, Nanocomposite hydrogels, Carbon nanotube, PVA/PVP hydrogels

\section{INTRODUCTION}

Controlled drug release systems have received much attention enabling the sustained release of the drugs at determined rates for required time periods [1-3]. These release systems offer good alternatives with high efficiency providing the reduced toxicity and increased patient compliance to avoid the disadvantages of the conventional dosage forms [4]. Hydrogels have been utilized in many biomedical applications including drug release with their high biocompatibility, significant swelling characteristics and 3-D macroporous network structure [5-7]. Poly(vinylalcohol) (PVA) hydrogels can be prepared via physical cross-linking with freeze-thawing process resulting a flexible, macroporous, stable and strong gel matrix [8,9]. In this method, there is no need to use any toxic chemicals such as cross-linkers and initiators. Thus physically-crosslinked PVA hydrogels present an excellent potential for the biomedical applications having high cytocompatibility [10]. Poly(vinylpyrolidone) (PVP) is another biomaterial that is widely used in biomedical applications due to its high biocompatibility and hydrophilic character [11]. The preparation of PVA/PVP hydrogels via freeze/thawing method and their various applications were reported elsewhere [12-16]. Recently, it has reached a tremendous importance in the development of novel systems with improved drug release profile and thus, hydrogel nanocomposites, which means the incorporation of the nanosized particles into the hydrogel matrix, have gained much interest since they enhance mechanical, physicochemical and drug release properties $[17,18]$. Carbon nanotubes $(\mathrm{CNT})$ are the most important components that are used in the composition of the hydrogel nanocomposites due to their remarkable mechanical, electrical and thermal properties and large surface area [19-21]. Also there have been many reports about the utilization of CNT as drug carriers indicating their potential for drug release systems with good biocompatibility [22-24]. Therefore the incorporation of CNT into the hydrogel structure has presented an attractive approach to develop CNT-based hydrogels as innovative drug delivery instruments [25].

*Corresponding Author: emeltamahkar@ hitit.edu.tr 
In this study, we report the preparation of CNT-based poly(vinylalcohol)/poly(vinylpyrolidone) (PVA/PVP) nanocomposite hydrogels for drug release applications. Firstly, these hydrogels were prepared via freeze/thawing method with different CNT compositions. In order to investigate the drug release profile of the hydrogels, 5-fluorouracil (FU) was selected as a model drug and was loaded into the nanocomposite hydrogel matrix. The nanocomposite hydrogels were characterized using swelling tests, SEM measurements, FTIR, DSC and BET analysis. The drug release mechanism was evaluated by fitting the experimental data to zero-order, first-order, Higuchi and Korsmeyer-Peppas kinetic model equations.

\section{MATERIALS AND METHODS}

\subsection{Materials}

Polyvinylalcohol (PVA) (Mw: 145000) was purchased from Merck. Polyvinylpyrrolidone (PVP) (Mw: 40000), multiwalled carbon nanotubes ( $10 \mathrm{~nm} \times 4.5 \mathrm{~nm}$ x $4 \mu \mathrm{m})$ (CNT) and dimethyl-sulfoxide (DMSO) were obtained from Sigma. Potassium chloride, sodium hydroxide, hydrochloric acid, potassium dihydrogen phosphate and sodium chloride were utilized for adjusting the phosphate buffer solutions to $\mathrm{pH} 7.4$ and all were obtained from Merck. The water used in the experiments was ultra-purified using Direct Q-3 purification system from Milipore (Molsheim France).

\subsection{Synthesis of CNT-Based PVA/PVP Hydrogels}

The CNT-based PVA/PVP hydrogels were synthesized using the same method described in our earlier publication for the preparation of PVA/PEG hydrogels via freeze/thawing [26]. Briefly, PVA was dissolved in distilled water to prepare $5 \%$ aqueous solution by using a magnetic stirrer for $2 \mathrm{~h}$ at $90{ }^{\circ} \mathrm{C}$ and then the solution was slowly cooled to room temperature. $5 \%$ solution of PVP was prepared at room temperature. Then, CNT was stirred in the solution of $\mathrm{H}_{2} \mathrm{O} / \mathrm{DMSO}(3: 1, \mathrm{v} / \mathrm{v})$ for $4 \mathrm{~h}$ [27]. The polymer solutions were mixed under magnetic stirring at room temperature for $2 \mathrm{~h}$. The mixture was placed on the 24-well plate. The blend solution was directly kept frozen at $-16^{\circ} \mathrm{C}$ for 16 hours. Afterwards, the frozen hydrogels were thawed at room temperature for 8 hours. This process of freeze/thawing was repeated for 5 times. Table 1 shows the feed composition for preparation of the hydrogels. To remove unreacted polymers, water changed with fresh water periodically for four days.

Table 1. The feed composition for preparation of the hydrogels

\begin{tabular}{llll}
\hline Code & PVA, $\%$ & PVP, $\%$ & CNT, mg \\
\hline PVA/PVP & 5 & 5 & - \\
\hline CNT-25 & 5 & 5 & 25 \\
\hline CNT-50 & 5 & 5 & 50 \\
\hline CNT-100 & 5 & 5 & 100 \\
\hline
\end{tabular}

\subsection{Characterization of CNT-based PVA/PVP hydrogels}

The swelling behavior of the dried samples were observed in $\mathrm{pH} 7.4$ buffer solution at $37^{\circ} \mathrm{C}$. A known disc samples were put into shaker water bath fixed at $50 \mathrm{rpm}$. The samples were taken out from the buffer solution, swollen hydrogels were filtered and weighed. The water content of the hydrogels were determined according to the following eqution:

$$
\text { Swelling } \%=\frac{W s-W d}{W_{d}} \times 100
$$

where $\mathrm{W}_{\mathrm{s}}$ and $\mathrm{W}_{\mathrm{d}}$ represent the weighs of swollen and dried state samples respectively. 
Synthezised hydrogels were also characterized using scanning electron microscopy (SEM) measurements with Quanta Feg 650 scanning electron microscope. The chemical structure was determined by Fourier transform infrared spectroscopy (FTIR) (FTIR 8000 Series, Shimadzu, Japan). The glass transition temperatures $\left(\mathrm{T}_{\mathrm{g}}\right)$ of the samples were identified using Differential Scanning Calorimetry (DSC) analysis. The experiments were performed using Shimadzu DSC-60H. The samples were heated at $10{ }^{\circ} \mathrm{C} / \mathrm{min}$ between $25^{\circ} \mathrm{C}$ and $160^{\circ} \mathrm{C}$ in nitrogen atmosphere. The specific surface area measurements were carried out by Brunauer-Emmitt-Teller (BET) method (Quaniochome, Autosorb IQ).

\subsection{Drug Loading and Release Behaviour}

To investigate the drug release behavior of the hydrogels, 5-FU was chosen and used as a model drug. The experiments of 5-FU loading onto the nanocomposite hydrogels were carried out in distilled water using $500 \mathrm{mg} / \mathrm{L}$ of 5 -FU aqueous solution for 2 days. Amount of drug loading was determined spectrophotometrically at $266 \mathrm{~nm}$ using Shimadzu UV-1800.

For release experiments, dried hydrogel discs containing 5-FU were placed in shaker water bath $20 \mathrm{~mL}$ buffer solutions $\mathrm{pH} 7.4$ at $37{ }^{\circ} \mathrm{C}$ and $50 \mathrm{rpm}$. At determined time, $0.5 \mathrm{~mL}$ of buffer solution was taken from the release medium, and $0.5 \mathrm{~mL}$ of buffer solution was put into the drug release medium.

$$
\text { Cumulative release }(\%)=\frac{\mathrm{C}_{\mathrm{n}} \mathrm{V}+\sum_{\mathrm{i}=1}^{\mathrm{i}=\mathrm{n}-1} \mathrm{C}_{\mathrm{i}} \mathrm{V}_{\mathrm{i}}}{\mathrm{m}} \times 100
$$

where $\mathrm{V}$ is the drug solution volume $(\mathrm{mL}), \mathrm{V}_{\mathrm{i}}$ is the sample volume $(\mathrm{mL}), \mathrm{m}$ is the hydrogel weight $(\mathrm{mg}), \mathrm{C}_{\mathrm{n}}$ and $\mathrm{C}_{\mathrm{i}}$ are the initial drug concentration and concentrations in the drug releasing medium at determined time interval respectively. All the data were repeated in triplicate.

\section{RESULTS AND DISCUSSION}

\subsection{Characterization}

To determine the effect of the amount of CNT on the swelling behavior of the hydrogels, the swelling characteristics of the nanocomposite hydrogels were investigated and listed in Table 2. The results demonstrate that the addition of CNT into the PVA/PVP hydrogels increased the swelling percentage with increasing the amount of CNT. The reason for this was caused by the incorporation of CNT into the polymeric matrix leading higher surface area. However, the water uptake of CNT-100 was lower than that of CNT-50 hydrogels since the distribution of CNT was heterogeneously distributed throughout the hydrogel matrix. These results are confirmed with the results reported elsewhere [21].

Table 2. The swelling percentages of PVA/PVP and CNT based hydrogels

\begin{tabular}{|l|l|}
\hline Polymer & \% Swelling \\
\hline PVA/PVP & $237.2 \pm 14.2$ \\
\hline CNT-25 & $241.9 \pm 15.5$ \\
\hline CNT-50 & $357.2 \pm 9.9$ \\
\hline CNT-100 & $272.6 \pm 12.6$ \\
\hline
\end{tabular}

The SEM images of PVA/PVP and CNT-based PVA/PVP hydrogels were demonstrated at Figure 1. It was seen that PVA/PVP hydrogels showed porous structure indicating the gelation via the freeze/thawing procedure. Figure 1.B showed the incorporation of CNT into the PVA/PVP hydrogels. CNT-25 hydrogels showed homogenously distributed and porous structure presenting a good alternative for the drug release carrier systems. CNT-50 hydrogels demonstrated homogenously distributed and porous morphological properties but a more smooth structure than CNT-25 hydrogels. However it was observed that in the CNT-100 hydrogels matrix structure non-homogenous accumulation resulting a 
non-uniform distribution of carbon nanotubes through the hydrogel matrix. Therefore CNT-50 hydrogels were selected to use for the further studies due to the proper morphological structure.
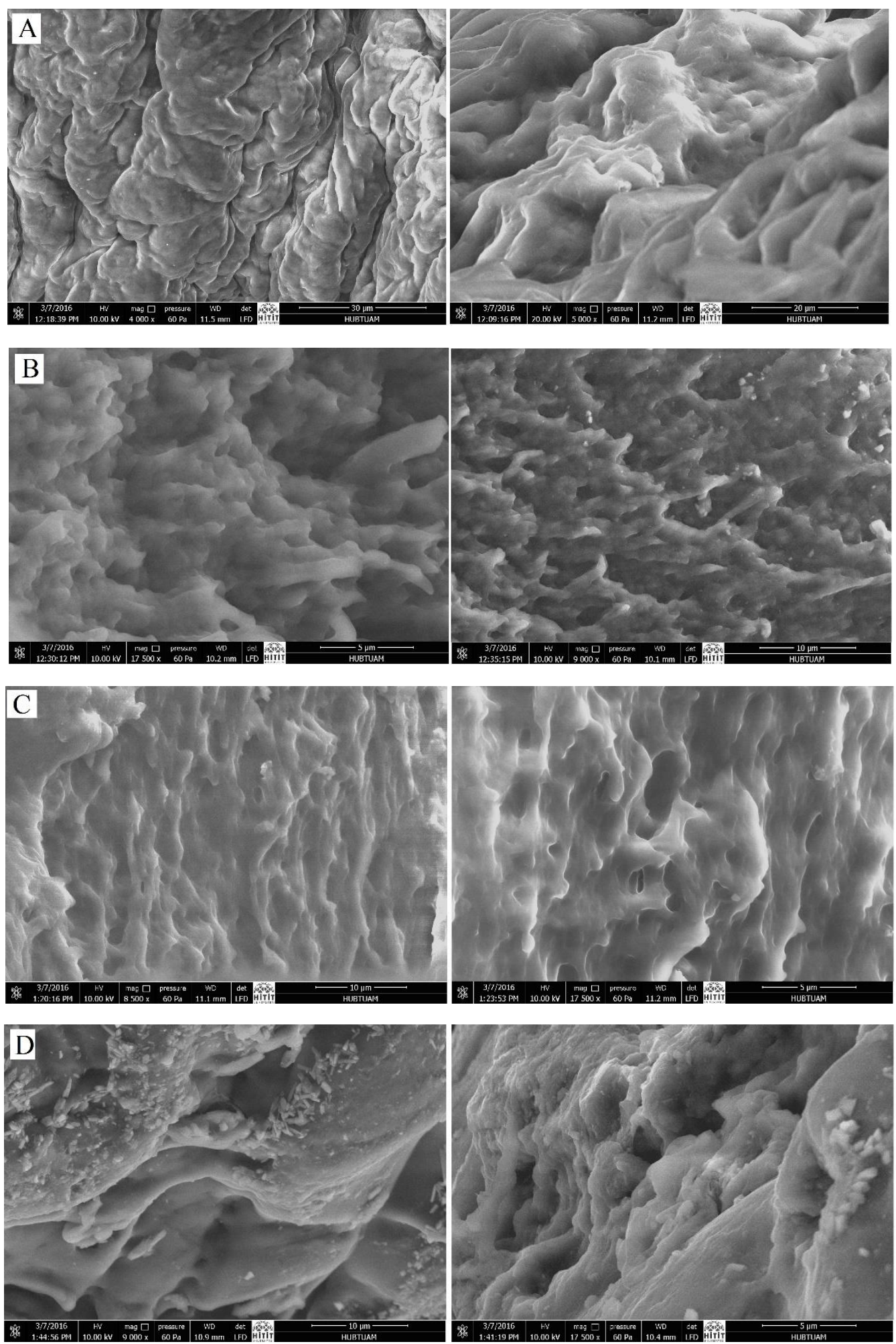

Figure 1. SEM images of PVA/PVP hydrogels. A.) PVA/PVP, B.) CNT-25 hydrogel, C.) CNT-50 hydrogel, D.) CNT-100 hydrogel. 
The FTIR spectrum of PVA/PVP and CNT-50 hydrogels were demonstrated in Figure 2. The intensity of the bands at around $3300 \mathrm{~cm}^{-1}$ and $2915 \mathrm{~cm}^{-1}$ was decreased with the addition of CNT to the polymeric structure. The intensity of the band at $1650 \mathrm{~cm}^{-1}$ was increased with the incorporation of CNT through the hydrogel matrix attributed to the formation of $\mathrm{C}-\mathrm{C}$ band between CNT and polymer chains. The new bands at $2941 \mathrm{~cm}^{-1}, 1420 \mathrm{~cm}^{-1}$ and $1138 \mathrm{~cm}^{-1}$ were appeared and the broad band at $1083 \mathrm{~cm}^{-1}$ was disappeared. All these changes in the spectrum was due to the presence of the CNT in the polymeric hydrogel structure [28].

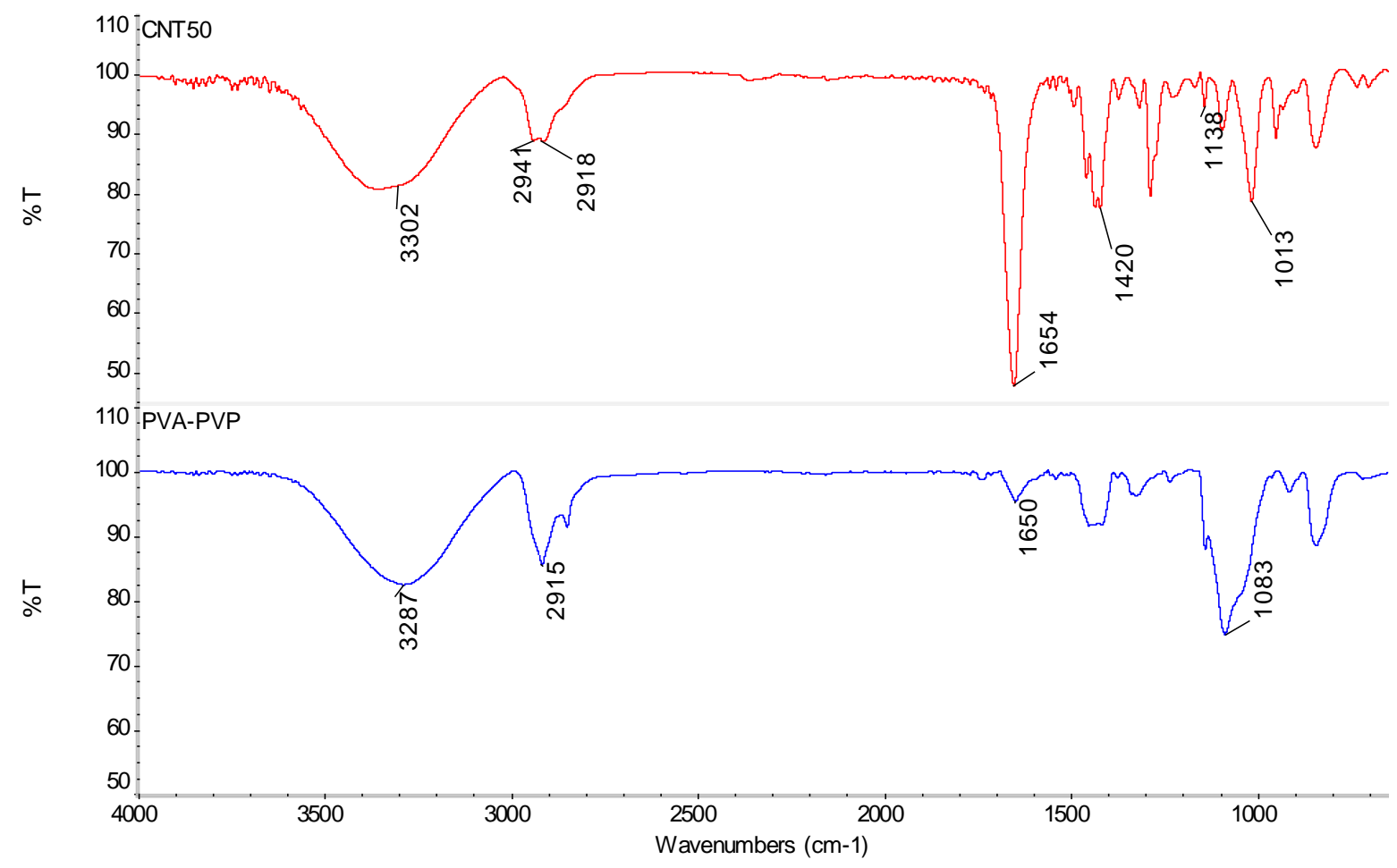

Figure 2. FTIR spectrum of PVA/PVP and CNT-50 hydrogels

DSC analysis is one of the most common methods for the determination of chemical structures of polymeric materials. Figure 3 shows the profiles of DSC curves of PVA/PVP hydrogel and CNT-50 hydrogel. It was observed a large melt peak at around $230{ }^{\circ} \mathrm{C}$ for PVA/PVP hydrogels and at around $225^{\circ} \mathrm{C}$ for CNT-50 hydrogels indicating the significant influence of CNTs for the crystallization of polymers. The reason for this is the increase in the crystallinity of the nanocomposite hydrogels where CNT acts as nucleation sites for the polymer-carbon nanotube interactions. The values of $\mathrm{T}_{\mathrm{g}}$ of PVA/PVP and CNT-50 hydrogels were calculated as $126.426{ }^{\circ} \mathrm{C}$ and $144.115{ }^{\circ} \mathrm{C}$ respectively. The results show that the incorporation of CNT was successfully achieved. 


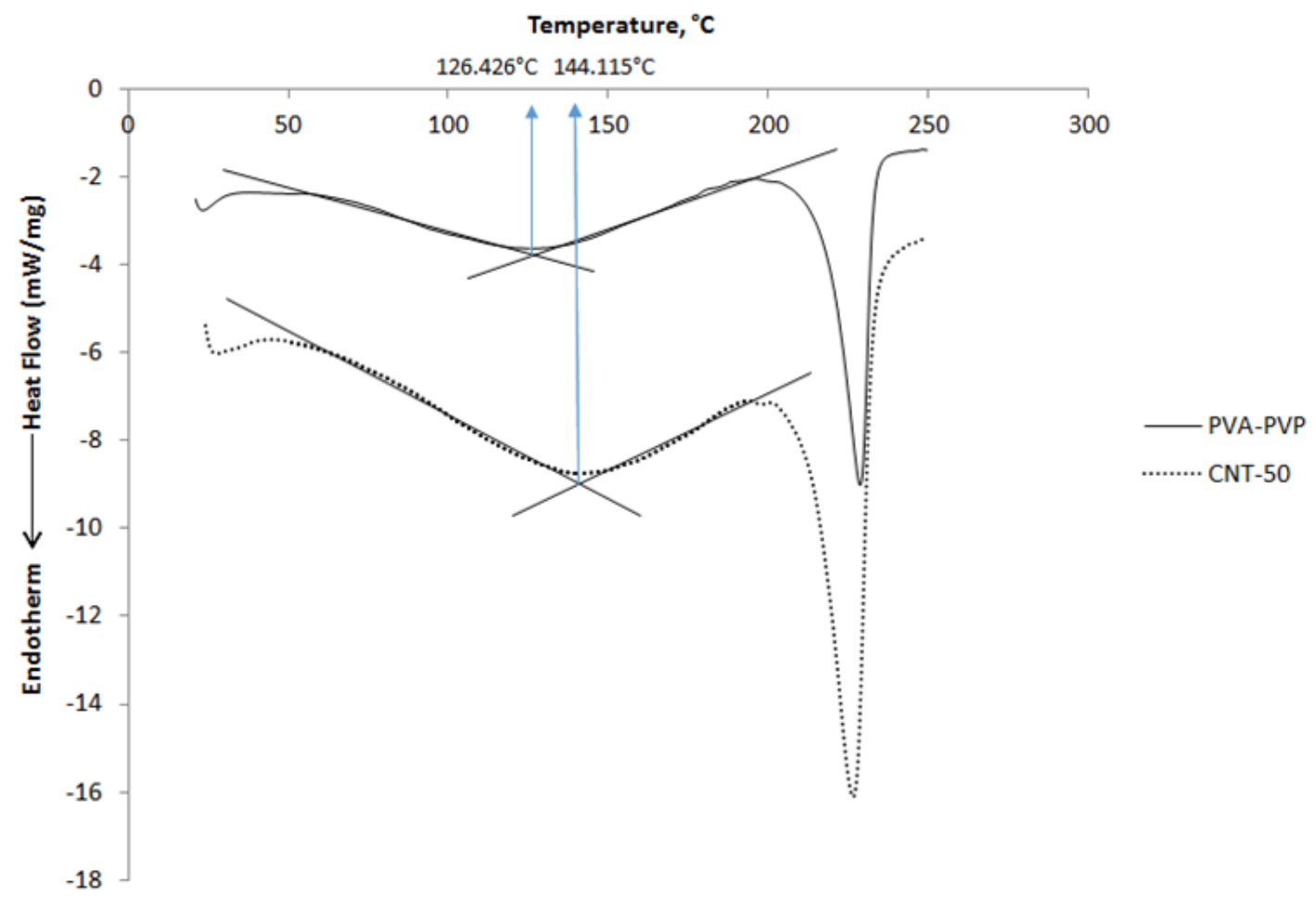

Figure 3. DSC curves of PVA/PVP and CNT-50 hydrogels

The large surface area is one of the important parameters for drug delivery applications. The incorporation of the nanomaterial is a general approach to enhance the surface area of the hydrogels. There have been many reports about the nanocomposite hydrogels with improved surface area $[29,30]$. In this study, it was determined due to the results of BET analysis that the surface area of CNT-50 hydrogel was increased by $42 \%$ with respect to PVA/PVP hydrogel with the incorporation of CNT to the hydrogel structure.

\subsection{Drug Loading and Release Tests}

The loading experiments of 5-FU as the model drug were performed ex-situ using the drug solution of $500 \mathrm{ppm}$ at room temperature. It was found that the loading efficiency of the 5-FU onto CNT-50 hydrogel was higher than that of PVA/PVP hydrogels. It was obtained that the presence of CNT increased 5-FU loading capacity via $\pi-\pi$ interactions. These results are in parallel with the results obtained from surface area measurements.

The in-vitro 5-FU release tests were performed at $37{ }^{\circ} \mathrm{C}$ at $\mathrm{pH}$ 7.4. The in-vitro release profiles were determined by plotting the cumulative release of the drug with respect to loaded amount of the drug. As shown in Figure 4, it was found that the cumulative released amount of 5-FU from the CNT-50 hydrogel (93\%) was higher than that of PVA/PVP hydrogel (43\%) after $144 \mathrm{~h}$. The release of 5-FU from the CNT based hydrogels could be fitted to 3 regions. In the first region that is between 1 and $6 \mathrm{~h}$, second region from $6 \mathrm{~h}$ to $72 \mathrm{~h}$ and the last region between $72 \mathrm{~h}$ and $144 \mathrm{~h}$. In the first region, initial burst release was observed with $38 \%$ of the drug was released from PVA/PVP hydrogels indicating the weak performance of this material for drug release applications. The cumulative release of 5-FU from CNT50 hydrogels was only $25 \%$ in this time period indicating the prevention of burst release of 5-FU by introducing of CNT into the hydrogel structure. In the second region, the prolonged and slow release of the drug from CNT-50 hydrogels was determined which attributes to the presence of CNT. In the last region, the cumulative release of 5-FU from both hydrogels remains constant. 


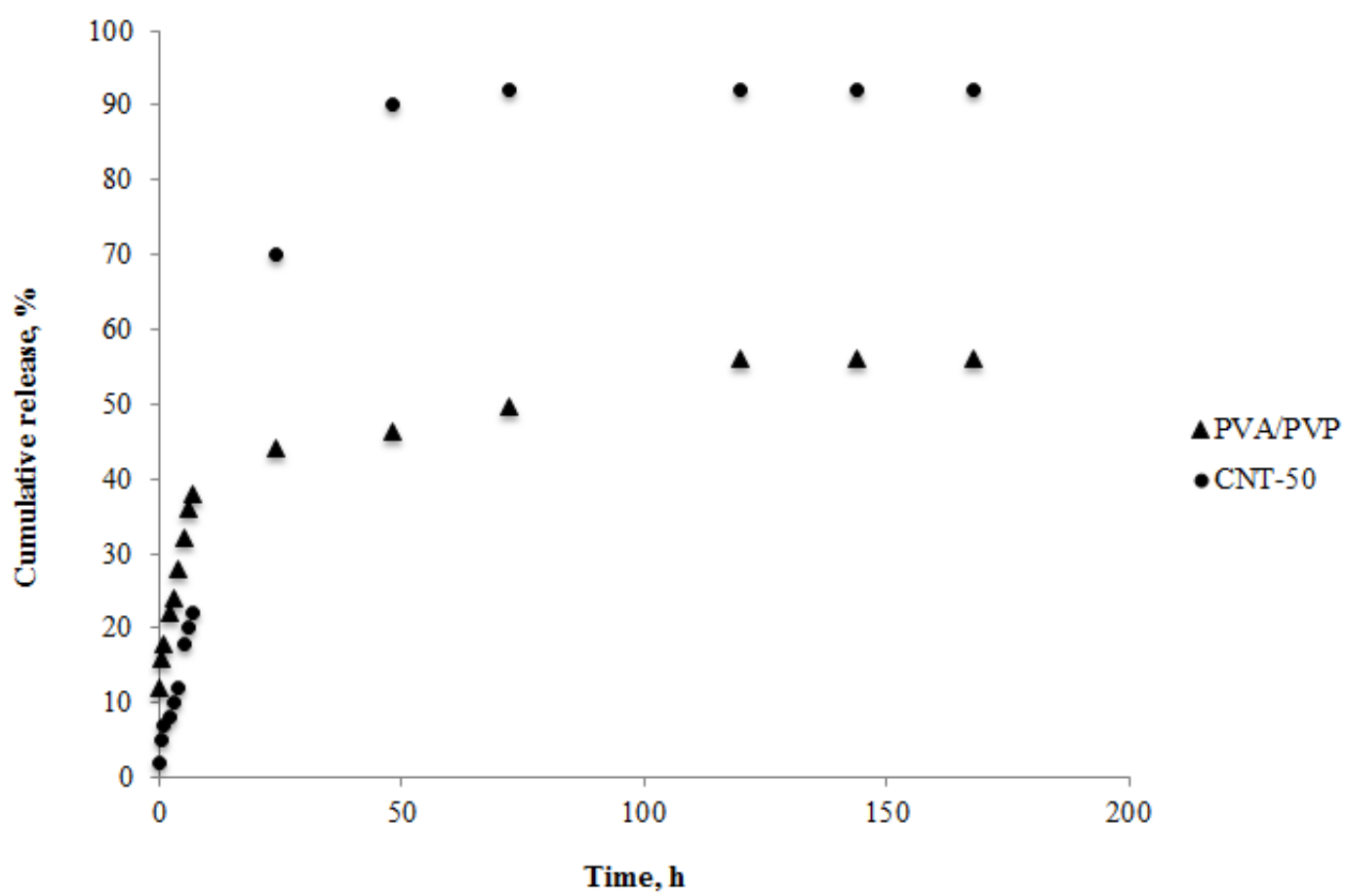

Figure 4. The release profiles of 5-FU of CNT-50 and PVA/PVP nanocomposite hydrogels

The comparison of percentage of cumulative release and release time of the drug delivery polymeric systems including CNT reported in the literature and listed in Table 3. The cumulative release percent of multi-walled CNT/PVA composites after $12 \mathrm{~h}$ was reported as $30 \%$. The composites were prepared via aqueous mixing and they wee used for the release of diltiazem for patch therapy [30]. PVA/PAA/multi-walled CNT nanofibers were prepared via electrospinning and utilized for the electroresponsive transdermal ketoprofen release. It was determined that the cumulative release percent was found as $90 \%$ after $10 \mathrm{~h} \mathrm{[31].} \mathrm{The} \mathrm{pH}$-responsive and electro-responsive microcapsules were developed with multi-walled CNT and PVA. After the composite microcapsules were modified using oxyfluorination, the fabrication of composite microcapsules was increased due to the hydrophilic functional groups. The cumulative drug release was defined as $80 \%$ after $30 \mathrm{~h}$ period [32]. It was reported that the cumulative release was achieved as $85 \%$ after $10 \mathrm{~h}$ with polyethylene oxide/pentaerythritol triacrylate/multi-walled CNT electrospun nanofibers [33]. It was seen that the percentage of cumulative release and release time obtained in this study are comparably higher than that of the results reported in the literature. Also it was determined that the controlled drug release of 5-FU was achieved with CNT-50 hydrogels indicating the strong interactions between drug molecules and composite hydrogels.

Table 3. The drug delivery polymeric systems including CNT reported in the literature

\begin{tabular}{|l|l|l|l|}
\hline Polymer content & Cumulative release percent, $\%$ & Release time, $\mathrm{h}$ & Reference number \\
\hline PVA & 30 & 12 & {$[31]$} \\
\hline PVA and PAA & 90 & 10 & {$[32]$} \\
\hline PVA and PAA & 80 & 30 & {$[33]$} \\
\hline PEO & 85 & 10 & {$[34]$} \\
\hline PVA and PVP & 93 & 144 & This study \\
\hline
\end{tabular}

PAA: polyacrylic acid, PEO: polyethylene oxide 


\subsection{Mathematical Modeling}

The 5-FU release mechanism from PVA/PVP and CNT-50 hydrogels was determined with 4 different release kinetic models, which are zero-order, first-order, Higuchi and Korsmeyer-Peppas kinetic models $[35,36]$. The zero-order kinetic model describes the systems, which are not related to the drug concentration while the first-order kinetic model defines the systems, which depend on drug concentration. Higuchi model proposes the correlation of drug release to square root of time and related with Fickian diffusion. Korsmeyer-Peppas kinetic model describes the drug release from swellingcontrolled systems. In this model, $\mathrm{n}$ indicates the information about release mechanism of the drug, which the drug release kinetics model fits to Fickian/diffusion model when $\mathrm{n}$ approximates to 0.5 , nonFickian model when $\mathrm{n}$ is between 0.5 and 0.85 and case II transport model when $\mathrm{n}$ is 1 respectively [37]. The release parameters of these kinetic models were listed in Table 4. It was seen obviously that Korsmeyer-Peppas model fitted the most to the both PVA/PVP and CNT-50 hydrogel systems due to the correlation coefficients of the applied kinetic models. It was observed that the 5-FU release from PVA/PVP hydrogels fitted to Fickian release behavior indicating that the dominant factor for drug release was swelling of the polymeric hydrogels. The release mechanism of 5-FU from CNT-50 hydrogels followed the non-Fickian release model indicating the 5-FU release was governed by the combination of diffusion of the drug and the dissolution of polymer chains.

The drug release profiles of PVA/PVP and CNT-50 hydrogels were evaluated using different mathematical drug release models, which are explained in detail below:

The zero order model is presented as:

$$
\mathrm{q}_{\mathrm{t}}=\mathrm{q}_{0}+\mathrm{k}_{0} \mathrm{t}
$$

where $\mathrm{q}_{\mathrm{t}}$ is the amount of drug released in time $\mathrm{t}, \mathrm{q}_{\mathrm{o}}$ is the initial amount of drug in the solution (usually $\left.\mathrm{q}_{\mathrm{o}}=0\right), \mathrm{k}_{\mathrm{o}}$ is the release rate constant of zero order kinetic model, and $\mathrm{t}$ is the drug release time.

The first order model is expressed as:

$$
\operatorname{In}\left(\mathrm{q}_{\mathrm{t}}\right)=\operatorname{In}\left(\mathrm{q}_{0}\right)-\mathrm{k}_{1} \mathrm{t}
$$

where $\mathrm{q}_{\mathrm{t}}$ is the amount of drug dissolved in time $\mathrm{t}, \mathrm{q}_{\mathrm{o}}$ is the initial amount of drug in the solution, and $\mathrm{k}_{1}$ is the first order release rate constant.

The Higuchi model is formulated as:

$$
\mathrm{q}_{\mathrm{t}}=\mathrm{k}_{\mathrm{H}} \sqrt{\mathrm{t}}
$$

where $\mathrm{q}_{\mathrm{t}}$ is the amount of drug released in time $\mathrm{t}$, and $\mathrm{k}_{\mathrm{H}}$ is the release rate constant of Higuchi kinetic model.

Korsmeyer-Peppas model is presented as:

$$
\mathrm{q}_{\mathrm{t}} / \mathrm{q}_{\infty}=\mathrm{k}_{\mathrm{k}} \mathrm{t}^{\mathrm{n}}
$$


Table 4. The release parameters of CNT-50 and PVA/PVP nanocomposite hydrogels

\begin{tabular}{|l|l|l|l|l|l|l|l|l|l|}
\hline \multirow{2}{*}{$\begin{array}{l}\text { Polymer } \\
\text { Code }\end{array}$} & \multicolumn{2}{|l|}{ Zero order equation } & \multicolumn{2}{l|}{ First order equation } & \multicolumn{2}{l|}{ Higuchi equation } & \multicolumn{3}{l|}{ Korsmeyer-Peppas equation } \\
\cline { 2 - 10 } & $\mathrm{k}_{0}$ & $\mathrm{R}^{2}$ & $\mathrm{k}_{1}$ & $\mathrm{R}^{2}$ & $\mathrm{k}_{\mathrm{H}}$ & $\mathrm{R}^{2}$ & $\mathrm{n}$ & $1.11 \mathrm{k}_{\mathrm{k}}$ & $\mathrm{R}^{2}$ \\
\hline $\begin{array}{l}\text { PVA/P } \\
\text { VP }\end{array}$ & 0.0150 & 0.472 & 0.0092 & 0.496 & 0.472 & 0.978 & 0.223 & 1.11 & 0.952 \\
\hline CNT-50 & 0.0438 & 0.732 & 0.0257 & 0.575 & 0.687 & 0.805 & 0.624 & 12.7 & 0.974 \\
\hline
\end{tabular}

\section{CONCLUSION}

The nanocomposite hydrogels have been gaining more attention with enhanced physicochemical characteristics for drug release systems. In this study, CNT-based PVA/PVP nanocomposite hydrogels were prepared via freeze/thawing method and their performances for drug release were also evaluated using 5-FU as a model drug. As regards to drug release behavior, CNT-based PVA/PVP nanocomposite hydrogels and PVA/PVP nanocomposite hydrogels were compared and the contribution of CNT to enhance the effect of controlled release of hydrogels was determined. It was found that KorsmeyerPeppas release kinetic model was fitted well to of CNT-based PVA/PVP nanocomposite hydrogels. The release mechanism of CNT-based PVA/PVP nanocomposite hydrogels was determined as non-fickian diffusion. It was concluded that the nanocomposite hydrogels prepared in this work offer great potential for drug release applications.

\section{REFERENCES}

[1] Serra L, Doménech J, Peppas NA. Drug transport mechanisms and release kinetics from molecularly designed poly(acrylic acid-g-ethylene glycol) hydrogels. Biomaterials 2006; 27: 5440-5451.

[2] Caccavo D, Cascone S, Lamberti G, Barba AA. Controlled drug release from hydrogel-based matrices: Experiments and modeling. Int J Pharma. 2015; 486: 144-152.

[3] Gentile P, Bellucci D, Sola A, Mattu C, Cannillo V, Ciardelli G. Composite scaffolds for controlled drug release: Role of the polyurethane nanoparticles on the physical properties and cell behaviour. $\mathrm{J}$ Mech Behav Biomed Mater 2015; 44: 53-60.

[4] Ambade AV, Savariar EN, Thayumanavan S. Dendrimeric micelles for controlled drug release and targeted delivery. Molecular Pharm 2005; 2: 264-272.

[5] Qiu Y, Park K. Environment-sensitive hydrogels for drug delivery. Adv Drug Del Rev 2001; 53: 321-339.

[6] Brandl F, Kastner F, Gschwind RM, Blunk T, Teßmar J, Göpferich A. Hydrogel-based drug delivery systems: Comparison of drug diffusivity and release kinetics. J Contr Rel 2010; 142: 221-228.

[7] Hoffman AS. Hydrogels for biomedical applications. Adv Drug Del Rev 2002; 54: 3-12.

[8] Jensen BEB, Dávila I, Zelikin AN. Poly(vinyl alcohol) physical hydrogels: Matrix-mediated drug delivery using spontaneously eroding substrate. J Phys Chem B 2016

[9] Qi X, Hu X, Wei W, Yu H, Li J, Zhang J, Dong W. Investigation of salecan/poly(vinyl alcohol) hydrogels prepared by freeze/thaw method. Carohydr Polym 2015; 118: 60-69.

[10] Hassan CM, Peppas NA. Structure and morphology of freeze/thawed PVA hydrogels. Macromol 2000; 33: 2472-2479. 
[11] Park KR, Nho YC. Preparation and characterization by radiation of poly(vinyl alcohol) and poly(Nvinylpyrrolidone) hydrogels containing aloe vera. J Appl Polym Sci 2003; 90: 1477-1485.

[12] Ma R, Xiong D, Miao F, Zhang J, Peng Y. Novel PVP/PVA hydrogels for articular cartilage replacement. Mater Sci Eng C 2009; 29: 1979-1983.

[13] Nho Y-C, Lim Y-M, Gwon H-J, Choi E-K. Preparation and characterization of PVA/PVP/glycerin/antibacterial agent hydrogels using $\gamma$-irradiation followed by freeze-thawing. Korean J Chem Eng 2009; 26: 1675-1678.

[14] Thomas J, Gomes K, Lowman A, Marcolongo M. The effect of dehydration history on PVA/PVP hydrogels for nucleus pulposus replacement. J Biomed Mater Res Part B Appl Biomater 2004; 69B: 135-140.

[15] Shi Y, Xiong D, Liu Y, Wang N, Zhao X. Swelling, mechanical and friction properties of PVA/PVP hydrogels after swelling in osmotic pressure solution. Mater Sci Eng C 2016; 65: 172-180.

[16] Shi Y, Xiong D. Microstructure and friction properties of PVA/PVP hydrogels for articular cartilage repair as function of polymerization degree and polymer concentration. Wear 2013; 305: 280-285.

[17] Shah K, Vasileva D, Karadaghy A, Zustiak SP. Development and characterization of polyethylene glycol-carbon nanotube hydrogel composite. J Mater Chem B 2015; 3: 7950-7962.

[18] Li Z, Tang M, Dai J, Wang T, Bai R. Effect of multiwalled carbon nanotube-grafted polymer brushes on the mechanical and swelling properties of polyacrylamide composite hydrogels. Polymer 2016; 85: 67-76.

[19] Serpell CJ, Kostarelos K, Davis BG. Can carbon nanotubes deliver on their promise in biology? Harnessing unique properties for unparalleled applications. ACS Central Science 2016; 2: 190-200.

[20]Huang Y, Zheng Y, Song W, Ma Y, Wu J, Fan L. Poly(vinyl pyrrolidone) wrapped multi-walled carbon nanotube/poly(vinyl alcohol) composite hydrogels. Composites Part A: Appl Sci Manufactur 2011; 42: 1398-1405.

[21] Tong X, Zheng J, Lu Y, Zhang Z, Cheng H. Swelling and mechanical behaviors of carbon nanotube/poly(vinyl alcohol) hybrid hydrogels. Mater Lett 2007; 61: 1704-1706.

[22] Bellingeri R, Alustiza F, Picco N, Acevedo D, Molina MA, Rivero R, Grosso C, Motta C, Barbero $\mathrm{C}$, Vivas A. In vitro toxicity evaluation of hydrogel-carbon nanotubes composites on intestinal cells. J Appl Polym Sci 2015; 132: n/a-n/a.

[23] Mehra NK, Palakurthi S. Interactions between carbon nanotubes and bioactives: A drug delivery perspective. Drug Discov Today 2016; 21: 585-597.

[24] Mandal B, Das D, Rameshbabu AP, Dhara S, Pal S. A biodegradable, biocompatible transdermal device derived from carboxymethyl cellulose and multi-walled carbon nanotubes for sustained release of diclofenac sodium. RSC Adv. 2016; 6: 19605-19611.

[25] Peng X, Zhuang Q, Peng D, Dong Q, Tan L, Jiao F, Liu L, Liu j, Zhao C, Wang X. Sustained release of naproxen in a new kind delivery system of carbon nanotubes hydrogel. Iran J Pharm Res 2013; 12: 581-586. 
[26] Tamahkar E, Özkahraman B. Potential evaluation of PVA-based hydrogels for biomedical applications. HJSE 2015; 2: 165-171.

[27] Bin Y, Mine M, Koganemaru A, Jiang X, Matsuo M. Morphology and mechanical and electrical properties of oriented PVA-VGCF and PVA-MWCNT composites. Polymer 2006; 47: 1308-1317.

[28] Alghunaim NS. Optimization and spectroscopic studies on carbon nanotubes/PVA nanocomposites. Results Phys 2016; 6: 456-460.

[29] Fan L, Zhang J, Wang A. In situ generation of sodium alginate/hydroxyapatite/halloysite nanotubes nanocomposite hydrogel beads as drug-controlled release matrices. J Mater Chem B 2013; 1: 62616270 .

[30] Giuseppe Cirillo, Silke Hampel, Umile Gianfranco Spizzirri, Ortensia Ilaria Parisi, Nevio Picci, Iemma F. Carbon nanotubes hybrid hydrogels in drug delivery: A perspective review. BioMed Research International 2014; 2014: 1-17.

[31] Bhunia T, Giri A, Nasim T, Chattopadhyay D, Bandyopadhyay A. A transdermal diltiazem hydrochloride delivery device using multi-walled carbon nanotube/poly(vinyl alcohol) composites. Carbon 2013; 52: 305-315.

[32] Yun J, Im JS, Lee Y-S, Kim H-I. Electro-responsive transdermal drug delivery behavior of PVA/PAA/MWCNT nanofibers. Eur Polym J 2011; 47: 1893-1902.

[33] Yun J, Im JS, Lee Y-S, Bae T-S, Lim Y-M, Kim H-I. PH and electro-responsive release behavior of MWCNT/PVA/PAAc composite microcapsules. Colloids Surf A 2010; 368: 23-30.

[34] Im JS, Bai BC, Lee Y-S. The effect of carbon nanotubes on drug delivery in an electro-sensitive transdermal drug delivery system. Biomaterials 2010; 31: 1414-1419.

[35] Zou X, Zhao X, Ye L, Wang Q, Li H. Preparation and drug release behavior of pH-responsive bovine serum albumin-loaded chitosan microspheres. J Ind Eng Chem 2015; 21: 1389-1397.

[36] Kong BJ, Kim A, Park SN. Properties and in vitro drug release of hyaluronic acid-hydroxyethyl cellulose hydrogels for transdermal delivery of isoliquiritigenin. Carohydr Polym 2016; 147: 473481.

[37] Vaghani SS, Patel MM, Satish CS. Synthesis and characterization of $\mathrm{pH}$-sensitive hydrogel composed of carboxymethyl chitosan for colon targeted delivery of ornidazole. Carbohydr Res 2012; 347: 76-82. 